\title{
COLOUR CONFINEMENT IN THE LATTICE LANDAU GAUGE QCD SIMULATION
}

\author{
Sadataka FURUI \\ School of Science and Engineering, Teikyo University, \\ E-mail: furui@liberal.umb.teikyo-u.ac.jp \\ Hideo NAKAJIMA \\ Department of Information Science, Utsunomiya University, \\ E-mail: nakajima@is.utsunomiya-u.ac.jp
}

\begin{abstract}
The colour confinement criterion proposed by Kugo and Ojima is tested in the lattice Landau gauge QCD simulation. The renormalization effects are studied by measuring the gluon propagator, ghost propagator, three gluon vertex and the ghost-antighost-gluon vertex. The running coupling $\alpha_{s}$ from ghost-antighost-gluon vertex in the infrared region yields $\Lambda_{\widetilde{M O M}} \simeq 1 \mathrm{GeV}$, consistent to that from three gluon vertex in high momentum region.
\end{abstract}

\section{The confinement signal and the definition of the gauge field}

Two decades ago, Gribov pointed out a possible mechanism of colour confinement in Coulomb gauge or Landau gauge QCD via infrared divergence of the Faddeev-Popov ghost propagatoll. At nearly the same time, Kugo and Ojima proposed a criterion for the absence of coloured massless asymptoptic states in Landau gauge QCD using the BRST symmetry.

We study the confinement signal in the gluon propagator, the ghost propagator and the Kugo-Ojima parameter, and their dependence on the gauge field. Usually, the gauge field on lattice $A_{\mu}(x)$ is defined from the link variable $U_{\mu}(x)$ as $A_{x, \mu}=\left.\frac{1}{2}\left(U_{x, \mu}-U_{x, \mu}^{\dagger}\right)\right|_{\text {traceless part }}$, which we call $U$-linear version. A more natural definition is $U_{x, \mu}=\exp A_{x, \mu}, \quad A_{x, \mu}^{\dagger}=-A_{x, \mu}$, which we call $\log U$ version.

We observe that the Kugo-Ojima parameter $u^{a b}(0)$ at $\beta=5.5$ is about -0.7 in the $\log U$ and about -0.6 in the $U$-linear version.

If the configuration is in the core regiond, the tensor $G_{\mu \nu}^{a b}=\operatorname{tr}\left(\bar{\lambda}^{a} D_{\mu} \frac{1}{-\partial D}\left(-D_{\nu}\right) \lambda^{b}\right)_{x y}$ divided by $N^{2}-1$, where $N$ is the number of colours is expected to approach a function $E(U)$ defined by the optimizing function 1 . They are $E(U)=$ $\sum_{l} \frac{1}{N} \operatorname{Re} \operatorname{tr} U_{l}$ in $U$-linear version, and $\frac{1}{N^{2}-1} \sum_{l, a} \operatorname{tr}\left(\lambda^{a \dagger} S\left(\mathcal{A}_{l}\right) \lambda^{a}\right)$, where $\mathcal{A}_{l}=$ $\operatorname{adj}_{A_{l}}$ and $S(x)=\frac{x / 2}{\operatorname{th}(x / 2)}$, in $\log U$ version.

In the table below $e_{1}$ and $e_{2}$ stand for $e=\left\langle\frac{E(U)}{V}\right\rangle$, in our $16^{4}$ lattice simulation of the $U$-linear and the $\log U$ version of the gauge fields, respectively. 


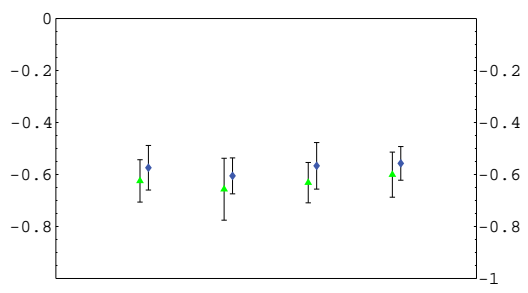

Figure 1: The dependence of the KugoOjima parameter measured along $\mathrm{x}, \mathrm{y}, \mathrm{z}$ and $\mathrm{t}$ axis resp. on the definition of the gauge field. $\beta=6.0,16^{4}$. The triangles are $\log U$, and diamonds are $U-$ linear version.

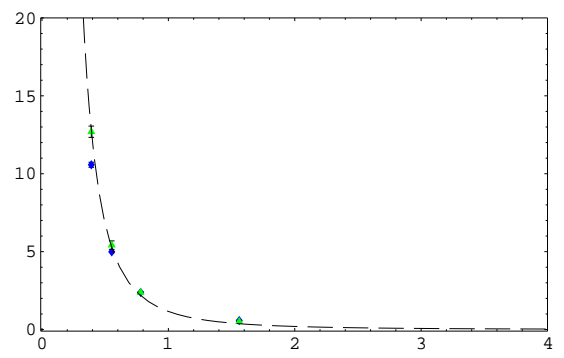

Figure 2: The ghost propagator as a function of the lattice momentum. $\beta=6.0,16^{4}$. Triangles are $\log U$ definition and diamonds are $U$-linear definition. The dashed curve is $1.162 / p^{2.545}$

We define the horizon function $h=\left\langle\frac{H(U)}{V}\right\rangle /\left(3\left(N^{2}-1\right)\right)=c-e / d$. In general we expect $h<0$ and in the continuum limit, we expect $h=0$ when the configuration is in the core region.

Table 1: $\beta$ dependence of the Kugo-Ojima parameter $c$, trace $e$ divided by the dimension $d$, and $h=c-e / d$. The suffix 1 corresponds to the $u$-linear and 2 corresponds to the $\log U$ version. Data are those of $16^{4}$, except $\beta=5.5 U$-linear data, which are those of $8^{4}$.

\begin{tabular}{c|ccc|ccc}
$\beta$ & $c_{1}$ & $e_{1} / d$ & $h$ & $c_{2}$ & $e_{2} / d$ & $h$ \\
\hline 5.5 & $0.570(58)$ & $0.780(3)$ & -0.21 & $0.712(18)$ & $0.908(1)$ & -0.20 \\
6.0 & $0.576(79)$ & $0.860(1)$ & -0.28 & $0.628(94)$ & $0.943(1)$ & -0.32 \\
\hline
\end{tabular}

The gluon propagator is infrared finite and the absolute value in $\log U$ version is about $20 \%$ larger than that in $U$-linear version The corresponding difference in the ghost propagator is about $10 \%$.

\section{The QCD running coupling}

The QCD running coupling $\alpha(\mu)=g^{2} / 4 \pi$ can be measured from the three gluon vertice 1 as, $g\left(\mu^{2}\right)=\frac{G_{A}{ }^{(3)}\left(p_{i}{ }^{2}, p_{f}{ }^{2}, p_{c}{ }^{2}\right) Z_{3}^{3 / 2}\left(\mu^{2}\right)}{G_{A}{ }^{(2)}\left(p_{i}{ }^{2}\right) G_{A}{ }^{(2)}\left(p_{f}{ }^{2}\right) G_{A}{ }^{(2)}\left(p_{c}{ }^{2}\right)}$. Since the lattice data of $G_{A}{ }^{(2)}$ is infrared finite, in contrast to the conjecture that the gluon propagator is infrared vanishingl, $g\left(\mu^{2}\right)$ decreases as $\mu$ decreases as $1.5 \mu$. This behaviour does not agree with the results of Dyson-Schwinger approacl 1 , which suggest that $\alpha_{s}(\mu)$ monotonically increases to a finite constant as $\mu$ goes to 0 . 

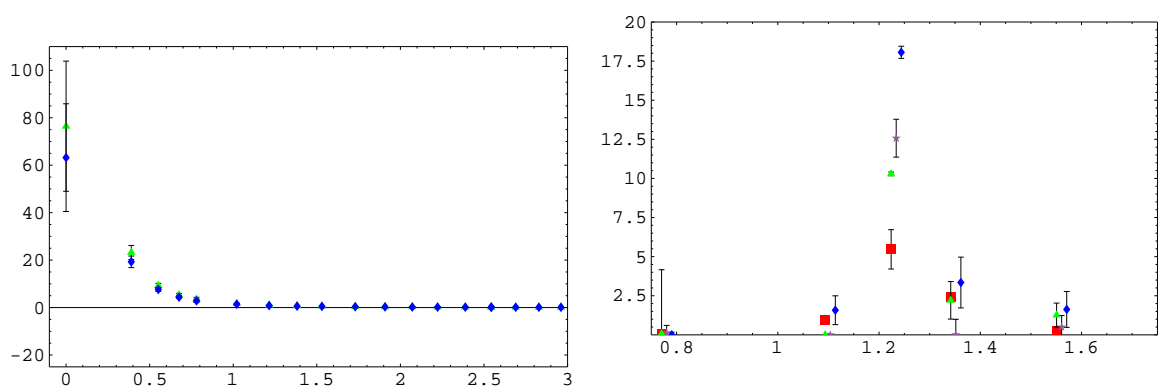

Figure 3: The gluon propagator as a function of the lattice momentum. $\beta=6.0,16^{4}$. Triangles are $\log U$ version and diamonds are $U$-linear version. 50 samples.

Figure 4: The QCD running coupling constant as a function of momentum $\mu$. Triangles and diamonds are $g^{2} / 4 \pi$, the same as Fig 3 , stars and boxes are $g^{2} / 4 \pi$ and $\tilde{g}^{2} / 4 \pi$, respectively of $\log U$ smeared version.

The running coupling can be obtained also from the ghost-antighost-gluon coupling $\tilde{g}\left(\mu^{2}\right)=\frac{G_{g}{ }^{(3)}\left(p_{i}{ }^{2}, p_{f}{ }^{2}, p_{c}{ }^{2}\right) Z_{3}^{1 / 2}\left(\mu^{2}\right) \tilde{Z}_{3}\left(\mu^{2}\right)}{G_{g}{ }^{(2)}\left(p_{i}{ }^{2}\right) G_{g}{ }^{(2)}\left(p_{f}{ }^{2}\right) G_{A}{ }^{(2)}\left(p_{c}{ }^{2}\right)}$. The preliminary results of $g^{2} / 4 \pi$ and $\tilde{g}^{2} / 4 \pi$ at symmetric momentum points $\left\langle p_{\mu}\right\rangle=\frac{2}{a} \sin \left(n_{\mu} \pi / L\right), \sum_{\mu} n_{\mu}^{2}=$ 10 have a peak which depend on definition of the $A_{\mu}$ but at other momentum points $g^{2} / 4 \pi$ and $\tilde{g}^{2} / 4 \pi$ are not so different. When the physical scale is fixed by $a^{-1}=1.91 \pm 0.1 \mathrm{GeV}, \Lambda_{\widetilde{M O M}}$ calculated from the $\tilde{g}^{2} / 4 \pi$ at $\sum_{\mu} n_{\mu}^{2}=8$ and 16 are about $1 \mathrm{GeV}$, which are consistent to that obtained from the three gluon vertex in high momentum regiorl.

S.F. thanks Prof. Reinhardt and Prof. Alkofer for helpful discussion and hospitality in Tuebingen in August 2000. This work is supported by JSPS Grant-in-aid No.11640251 and the KEK supercomputer project No.00-57.

\section{References}

1. T. Kugo and I. Ojima, Prog. Theor. Phys. Supp. 66, 1 (1979).

2. H.Nakajima and S. Furui, Nucl. Phys.B (Proc Suppl.)63A-C,635, 865(1999), idem, Nucl. Phys.B (Proc Suppl.)83-84,521 (2000); heplat/0004023, hep-lat/0006002, hep-lat/0007001.

3. V.N. Gribov, Nucl. Phys. B 139, 1 (1978).

4. D. Zwanziger, Nucl. Phys. B 364, 127 (1991), idem B 412, 657 (1994).

5. L. von Smekal, A. Hauck, R. Alkofer, Ann. Phys.267,1 (1998), hepph/9707327; R. Alkofer and L. von Smekal, hep-ph/0007355.

6. B. Allés et al., Nucl. Phys. B 502, 325 (1997); D. Henty et al.,EPS HEP 1995 239(1996);Ph. Boucaud et al., hep-ph/9810322,9810437 